\title{
125 years of European Neurology
}

The history of European Neurology begins in 1897 when the journal first appeared as Monatsschrift für Psychiatrie und Neurologie (Fig. 1). European Neurology (Fig. 2), together with Psychopathology, is the fourth oldest journal launched by Samuel Karger, and its history confounds with the history of the publishing house.

The journal was founded by the German Doctors C. Wernicke and T. Ziehen. The idea was to establish a journal to discuss and expand on the study of nerves and mental illness. In 1897 this area of medicine was poorly explored but progressing. The topic was in the foreground of the medical interest, and a new journal focused on this field of knowledge was necessary and justified [1]. Back then, the publication was released monthly, and the published content was in the German language only. Monatsschrift für Psychiatrie und Neurologie was published until 1957, when the periodical was renamed as Psychiatria et Neurologia (Fig. 3). In 1957, the name change came as a need to restructure the publication to meet a broader market and allow internationalization. This initiative also enabled the collaboration with editors from other European countries, and it opened the journal to publish reports in either German, English, French, or Italian with summaries in each of the other languages [2].

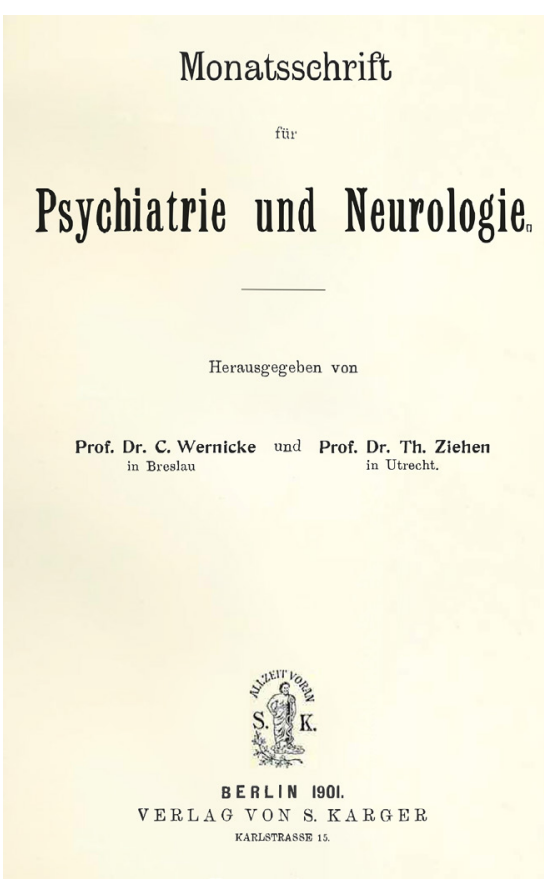

Fig.1. Image of the cover of Monatsschrift für Psychiatrie und Neurologie. 


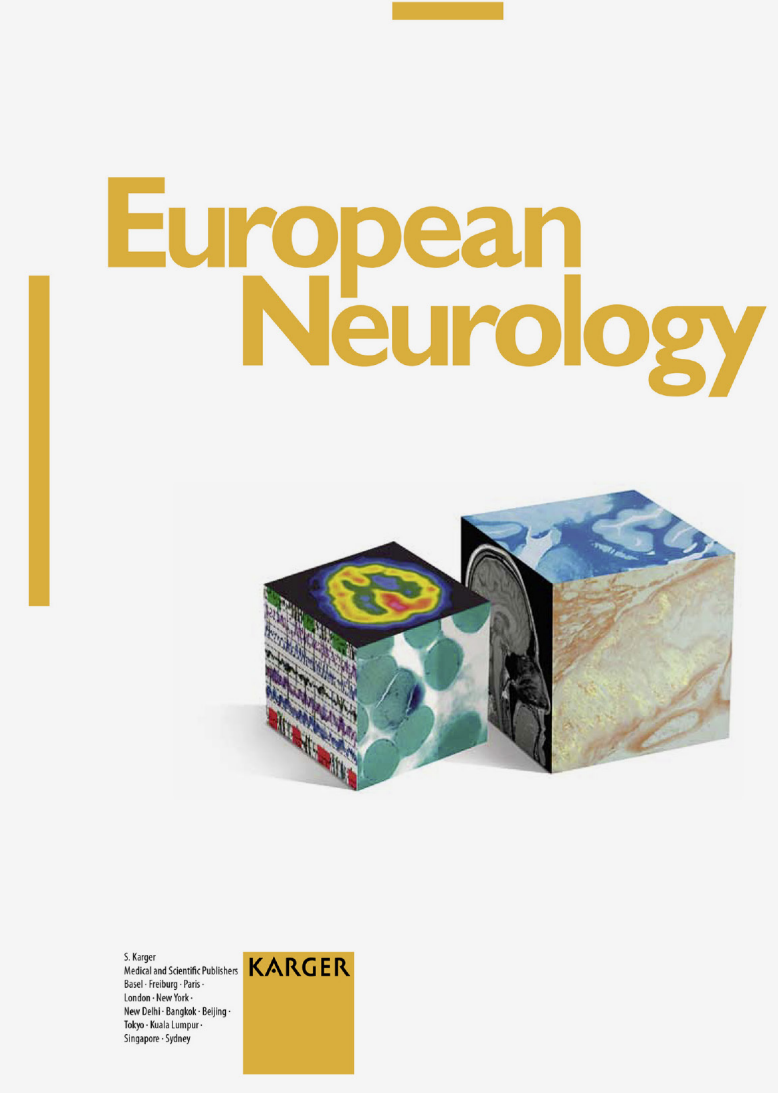

Fig. 2. Images of two different cover designs of European Neurology.

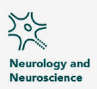

\section{European Neurology}

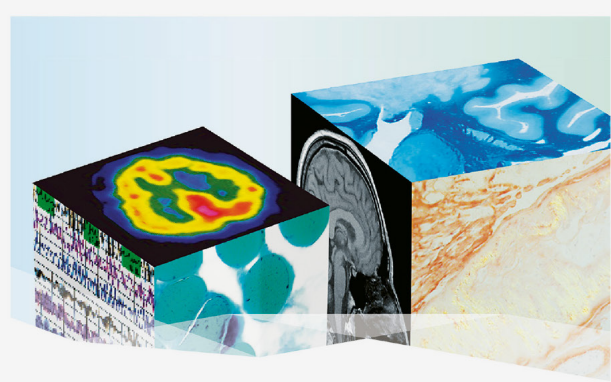

RESEARCH

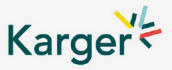

Once again, in 1968, the publication went through a radical change to keep pace with the scientific developments in the field. Under the leadership of H.E. Kaeser, the publication was split into two independent journals, and while the psychiatry reports would be published under the title Psychiatria Clinica (Fig. 4) (today Psychopathology), the neurological section of the journal continued to be published in European Neurology. This division was in reflection of the fact that after 70 years since the journal's foundation, basic science and clinical research have evolved into independence, and keeping the publication of reports of the two disciplines in the same media was artificial and impeding the progress in the communication of the developments in either field [3]. Also, in 1968 it was decided that European Neurology would publish exclusively in English, and besides serving as a vehicle for global dissemination and discussion of the important developments in neurology, it was also designed to provide an anglophone medium for the European neurologists, who encountered at the time great difficulties in

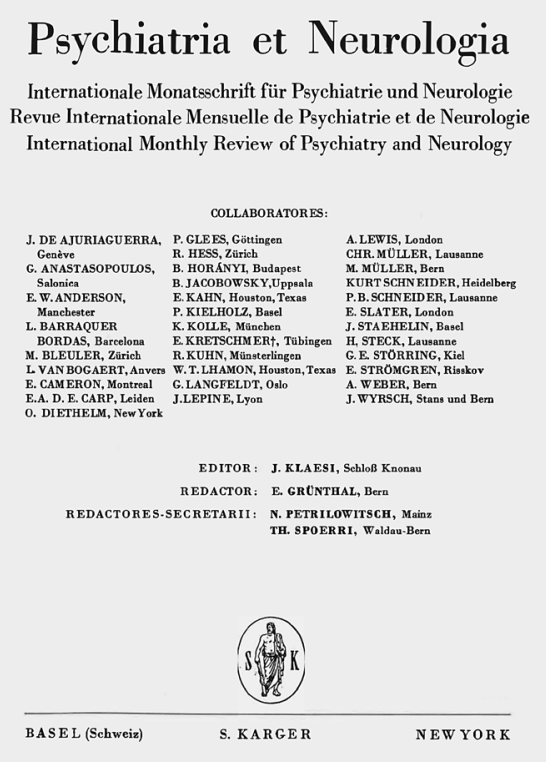

Fig. 3. Image of the cover of Psychiatria et Neurologia in 1957 showing the title in three different languages. 


\section{Psychiatria Clinica}

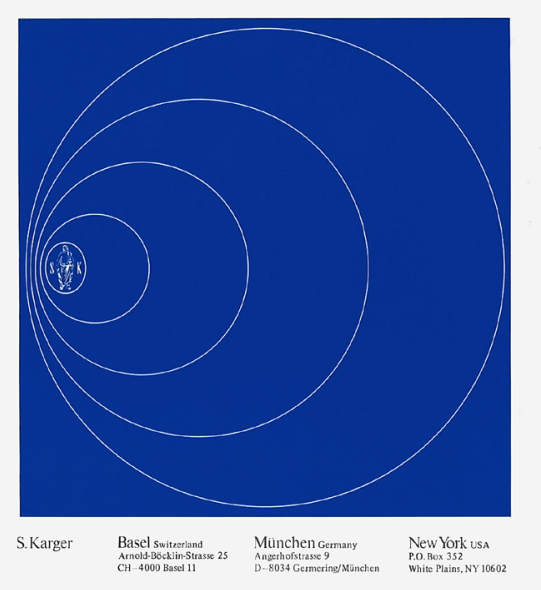

Fig. 4. Image of the cover of Psychiatria Clinica in 1968.

publishing in American and English journals [3]. While focusing on the specialty, the idea was to cross national and language boundaries and reaffirm the journal as an international periodical at the service of the broad neurological community.

A role we believe we have so far been able to play well. European Neurology has fundamentally contributed for 125 years as a source of quality research findings from renowned international scientists, clinicians, and early career researchers. Since 1968, we have published more than 5,790 articles in 84 volumes with contributions from co-authors across circa 66 countries that span out the European borders.

After more than a century, European Neurology is today an established and worldwide recognized journal in neurology. In observance of this milestone, in 2022, we will publish specially invited papers in a commemoratory anniversary section that will focus on the development of the treatments in neurology and their future. The celebratory section will be published with free access to reach a broad audience. All issues published in the jubilee volume will feature a specially created logo on their cover.

The current success of the publication is much owed to the leadership of Prof. J. Bogousslavsky, European Neurology's Editor-in-Chief since 1994, and his editorial team, whom we would like to thank for their continued commitment to publishing relevant findings and assuring the authorship with quick decisions and turnarounds. We would also like to thank our authors, reviewers, readers, and staff, for their support.

Gabriella Karger, Chairwoman and Publisher Daniel Ebneter, CEO

\section{References}

1. Wernicke C. I. Tagesfragen. Monatsschr Psychiatr Neurol. 1897;1:1-5.

2. Karger T. A short history of the Karger publishing firm. Bull Med Library Assoc. 1981;69:254-297.

3. Kaeser HE. Editorial. Eur Neurol. 1968;1:1. 